\title{
Distinct inflammatory gene expression in extraocular muscle and fat from patients with Graves' orbitopathy
}

\author{
Ivana Lopes Romero-Kusabara', José Vital Filho', Nilza Maria Scalissi², \\ Keli Cardoso Melo ${ }^{4}$, Giovanni Demartino ${ }^{4}$, Carlos Alberto Longui ${ }^{3,5}$, \\ Murilo Rezende Melo ${ }^{5}$ and Adriano Namo Cury ${ }^{2}$
}

${ }^{1}$ Department of Ophthalmology, ${ }^{2}$ Endocrinology Unit, Department of Medicine, ${ }^{3}$ Discipline of Pediatrics Endocrinology, Department of Pediatrics, Irmandade da Santa Casa de Misericórdia de São Paulo, São Paulo, Brazil, ${ }^{4}$ School of Medicine, and ${ }^{5}$ Molecular Medicine Laboratory, Department of Physiology, Santa Casa de Sao Paulo School of Medical Sciences, São Paulo, Brazil
Correspondence should be addressed to A N Cury

Email

anamo_cury@hotmail.com

\begin{abstract}
Objective: This study sought to compare patients with thyroid eye disease (TED) and normal controls with respect to the expression of the NR3C1, CHUK, IKBKB, FOS, NFKB and HSD11B1 genes in orbital fat (OF) and extraocular muscle (EOM).

Design and methods: A prospective study design was used to evaluate 34 TED patients and 38 healthy controls. OF was harvested from 33 TED patients and 27 controls. EOM biopsies were obtained from 32 TED patients and 18 controls. Samples were examined by real-time PCR and evaluated using appropriate statistical analyses with a significance cut-off of $P<0.05$.

Results: NR3C1 mRNA levels were higher in TED EOM (median 213 (96-376)) than those in control EOM (78 (34-138)) $(P<0.001)$, and NFKB expression was elevated in TED muscle (223 (31-520)) relative to that in control muscle $(8(6-31))(P<0.001)$. HSD11B1 expression was higher in TED EOM $(0.78(0.47-2.01))$ than that in control EOM (0.22 (0.09-0.51)) $(P<0.001)$. Levels of CHUK, IKBKB, and FOS were higher in TED EOM (115 (20-223), 111 (54-299) and 0.11 (0.03-0.19), respectively) than those in control EOM (5.8 (2-13), 21 (5-52) and 0.05 (0.001-0.03) respectively) $(P<0.001)$.

Conclusion: Tissues involved in GO exhibited different mRNA levels of NR3C1, CHUK, IKBKB, FOS, NFKB and HSD11B1. Gene expression in OF was similar for TED patients and controls. CHUK, IKBKB, FOS, NFKB, and HSD11B1 mRNA levels were higher in TED EOM than those in control EOM. NFKB was disproportionally elevated compared with NR3C1; this finding was indicative of a local proinflammatory profile.

\section{Introduction}

Graves' orbitopathy (GO) is an organ-specific autoimmune disease that is characterized by increased extraocular musculature, connective tissue of the orbit and adipose tissue $(1,2)$. GO affects $25-50 \%$ of Graves' disease patients and is the most significant extrathyroidal manifestation of this condition $(3,4)$.
(ㄷ) 2017 European Society of Endocrinology Printed in Great Britain
GO is caused by inflammation in the orbital tissues and fibroblast proliferation secondary to abnormal cellular autoimmune responses characterized by CD4+ and CD8+ $\mathrm{T}$ cell infiltration and expansion $(5,6)$ and excessive production of IL-1, IL-6, RANTES, IL-8, interferon- $\gamma$ and TNF- $\alpha$, which promote fibroblast proliferation and

Published by Bioscientifica Ltd. 
differentiation (7). There are phenotypic and functional differences between fibroblasts of orbital muscle and adipose tissues $(8,9)$.

The phenotypic difference between fibroblast subpopulations is based on the expression of the surface glycoprotein Thy-1 (CD90) (8). Thy-1-overexpressing orbital tissue induces fibroblast secretion of the cytokines IL-4, IL-5, IL-10 and TNF- $\alpha$; prostaglandin E2; hyaluronan and other hydrophilic substances such as glycosaminoglycan $(10,11)$. Adipogenesis is induced by cigarette smoke and prostaglandin D2 (secreted from active $\mathrm{T}$ cells), which drives the differentiation of peroxisomal proliferator-activated receptor- $\gamma$ (PPAR- $\gamma$ ) and IL-1 (12).

Depending on the exposure to TGF- $\beta$, fibroblasts can differentiate into adipocytes or muscle cells. Fibroblasts in the EOM are primarily Thy- 1 positive, and most fibroblasts in adipose tissue are Thy- 1 negative and are poised to become mature fat cells $(13,14,15)$. Thus, based on the inflammation profile, the timeline of the disease and the presence of factors such as CD90, orbital disease is categorized as having either a muscle or a fat predominance.

GO progresses through several stages of inflammation, first with a typical Th1 response and later with a Th2 response (16). The dynamics of the autoimmune response and the predominant cytokine profile determine the proliferation of fibroblasts and adipogenesis vs ongoing and progressive fibrosis, which is characterized by increased numbers of myofibroblasts. Recent findings have indicated that Th17 cells are involved in the active and/or fibrotic phases of orbital disease (17); such cells are $\mathrm{T}$ cells that produce IL-17A, which promotes inflammatory cytokines and extracellular matrix accumulation (18).

Therefore, other immune factors are likely to be involved in these processes, such as glucocorticoid receptor (NR3C1), nuclear transcription factor-kB (NFKB), inhibitor of $\mathrm{kB}(I K B K B)$, inhibitor of kappaB kinase (CHUK) and activator protein-1 (FOS), which mediate proinflammatory responses and cell growth, proliferation and survival $(19,20,21)$.

There are several mechanisms of NFKB activation. In the canonical pathway, NFKB is sequestered in the cytoplasm by IKBKB $(22,23)$. When cells receive a proinflammatory signal, the IKK complex activates and phosphorylates IKBKB, promoting degradation of the complex. After release from its cytoplasmic constraints, NFKB translocates into the nucleus, where it binds to specific target sequences. Thus, the activation of NFKB and FOS induce the transcription of cytokines, chemokines, adhesion molecules, enzymes and other inflammatory mediators $(24,25)$. NR3C1 is also likely to be involved in GO by decreasing the production and release of proinflammatory cytokines comprising primarily TNF- $\alpha$, IL-1, IL-2 and IL-6 $(26,27)$.

The study by Tomilson et al. (28) examined the influence of HSD11B1 and NR3C1 on adipogenesis in GO patients and reported higher mRNA expression levels in fat tissue. Their main objective was to demonstrate that HSD11B1 activity and cortisol bioavailability are crucial for the differentiation and proliferation of preadipocytes and de novo adipogenesis depending on the inflammatory profile in the orbital microenvironment. In that study, muscle tissue expression of $N F K B, C H U K, I K B K B, N R 3 C 1$ and $H S D 11 B 1$ were not reported.

In the present study, we examined the expression of NR3C1, CHUK, IKBKB, NFKB, FOS and HSD11B1 in both orbital fat (OF) and extraocular muscle of patients with GO.

\section{Subjects and methods}

\section{Sample and selection criteria}

The sample group comprised 34 individuals with GO and 38 controls without Graves' disease or ocular inflammatory disease who were evaluated from March 2010 to November 2013.

\section{Selection of patients with GO}

Patients in the GO group satisfied the diagnostic criteria for GO established by (29). These criteria specify the presence of palpebral retraction accompanied by not only thyroid dysfunction detected by the measurement of TSH, free T4, T3, total T4 and TRAb but also exophthalmia (defined as a Hertel exophthalmometry measurement $\geq 20 \mathrm{~mm}$ as the majority of patients were Caucasian), optic nerve dysfunction or extraocular muscle involvement, such as via restrictive myopathy or an increase in extraocular muscle, as revealed by orbital computed tomography.

All the candidates in the GO group underwent a complete ophthalmological evaluation and were classified based on disease activity according to the CAS (clinical activity score) and NOSPECS (Werner's classification) $(30,31)$. The current grading systems used to assess GO are the VISA (vision, inflammation, strabismus and appearance) and European Group of Graves' Orbitopathy (EUGOGO) classification systems. 
Both systems are grounded in the NOSPECS and CAS classification approaches and use indicators to assess signs of activity and degrees of severity. Coronal and axial computerized tomography of the orbits was performed to evaluate the orbitopathy and to plan for surgery.

We selected individuals who were classified as Werner 3,4 and 5, which is indicative of surgical decompression of the orbit, and with a CAS of 0 to 2 (i.e. those without clinically active disease and not requiring corticotherapy prior to surgery). During the orbit decompression procedure, EOM and OF samples were collected.

\section{Subclassification of the GO group}

GO individuals with a CAS $\leq 2$ (fibrotic orbitopathy) were subdivided into subtype 1 , which was predominantly lipogenic without diplopia, and subtype 2, which was predominantly myogenic with restricted extraocular motility and diplopia within $20^{\circ}$ of the primary gaze on clinical examination according to Nunery's classification. All subtype 2 patients showed an increase in the extraocular musculature on tomography.

\section{Selection of control patients}

Controls were recruited spontaneously and fulfilled the following requirements upon examination: negative family history (first-degree kin) for GD or other autoimmune thyroid diseases, TSH and free T4 levels in the normal reference range, no diagnostic criteria for $\mathrm{GO}$ and positive indications for an ocular surgical procedure such as evisceration due to painful blind eye, planned elective blepharoplasty or correction of strabismus.

The control group comprised 11 patients who had undergone evisceration of a painful blind eye, from whom muscle (right lateral) and fat were obtained; 20 blepharoplasty patients, from whom fat was collected and 7 patients who had undergone correction of strabismus, from whom only muscle was collected.

\section{Exclusion criteria}

The exclusion criteria were glucocorticoid use during the preceding 3 months, prepubertal status, an uncertain diagnosis and an ocular inflammatory condition or history of chronic inflammatory disease.

\section{Ethical approval}

This prospective study was conducted after approval by the institutional ethics committee. Signed informed consent was obtained from all patients.

\section{Laboratory methods}

\section{RNA extraction}

The fat and muscle biopsy samples were placed in 2-mL Eppendorf tubes, containing $750 \mu \mathrm{L}$ RNA Later (AMBION) and stored at $-80^{\circ} \mathrm{C}$. RNA was extracted using the RNeasy Lipid Tissue Mini Kit (Qiagen) following the manufacturer's instructions.

\section{Reverse transcription}

RNA was diluted in $50 \mu \mathrm{L}$ GIBCO ultrapure water (Life Technologies catalog\# $\mathrm{N}$ 8080234) and subjected to reverse transcription using TaqMan Reverse Transcription Reagents.

\section{Real-time $P C R$}

NR3C1, CHUK, IKBKB, FOS, NFKB and HSD11B1 mRNA levels were measured by real-time PCR using an ABI 7500 system (Applied Biosystems). All reactions were performed in duplicate with specific primers that had previously been standardized by our group. Values are presented as expression units and were normalized relative to the expression of the control gene $B C R$.

\section{Statistical analysis}

Statistical analyses were performed using Minitab 15 (Minitab, State College, PA, USA) and SigmaStat v3.5 (SPSS), and $P<0.05$ was considered significant. Categorical and qualitative variables were compared using a $\chi^{2}$ test and Fisher's exact test respectively. Continuous variables between groups were compared using the $t$-test or the Mann-Whitney test, depending on the normality of the data distribution as determined by the Kolmogorov-Smirnov test. The relationship between 2 continuous variables was established using the Pearson test. Outliers were defined by a Cook's distance $>4$. 


\section{Results}

The mean \pm S.D. age of the patients was similar between groups $(47.3 \pm 11.5$ years in the GO group and $50.4 \pm 16.3$ years in the control group) $(P=0.362)$, as was the gender distribution $(P=0.393)$. The mean \pm S.D. age of the type 2 GO patients $(50.4 \pm 10$ years) was higher than that of the for type 1 patients $(41.1 \pm 12$ years $)(P=0.026)$. The female:male ratio observed in GO type 1 lipogenic and GO type 2 myogenic patients were 70:30\% and 40:60\% respectively. Despite the proportion of males seems to be higher in type 2 myogenic group, statistical analyses did not reach significance (Z-test of two proportions; $P=0.228)$.

The median duration (months) of follow-up from the diagnosis of GO for subtypes 1 (23, 16.25-38.50) and $2(25,13.25-46.5)$ did not differ $(P=0.659)$. During clinical follow-up, $41 \%$ of the patients were in subclinical hyperthyroidism, 14\% were in clinical hyperthyroidism, $31 \%$ in euthyroidism and $14 \%$ in biochemical hypothyroidism at the last evaluation before the orbital decompression. In all cases, adjustments were made in the antithyroid drug or in the levothyroxine dosage when hypothyroidism resulted from a thyroidectomy procedure or after radioiodine therapy.

All patients in hyperthyroidism were treated with antithyroid drugs as the first line of treatment. Eleven (33\%) patients underwent definitive treatment with radioiodine therapy and the time between the dose of radioiodine and the surgical procedure of the orbit was 4 (12\%) of the patients underwent thyroidectomy, 17 (55\%) patients were treated exclusively with antithyroid drugs.

NR3C1, CHUK, IKBKB, FOS, NFKB and HSD11B1 expression levels in muscle tissue were elevated in the GO group vs the control group. The expression levels did not differ in OF tissue (Table 1).

The expression ratio of $N R 3 C 1 / N F K B$ in OF was similar in the two groups, but it decreased in the EOM of the GO group because of an increase in NFKB expression in the muscle tissue of GO patients (Table 2).

A similar expression ratio of NR3C1/HSD11B1 in the EOM and fat tissue was observed in GO patients compared to controls (Table 2).

We also tested whether the Nunery classification (GO subtypes 1 and 2) correlated to a specific expression profile of NR3C1, CHUK, IKBKB, FOS, NFKB and HSD11B1. In muscle and fat tissue, there were no significant differences with regard to the GO subtype, excluding FOS expression, which was increased in muscle from GO patients compared with controls (Table 3).
Table 1 Expression of NR3C1, NFKB, HSD11B1, FOS, IKBKB and $C H U K$ determined by real-time PCR in muscle tissue and ocular fat in GO patients and controls. Results are presented as median (interquartile interval) by Mann-Whitney test.

\begin{tabular}{|c|c|c|c|}
\hline Gene & GO & Controls & $\boldsymbol{P}$ \\
\hline \multicolumn{4}{|l|}{ Muscle } \\
\hline NR3C1 & $213(96-376)$ & $78(34-138)$ & $<0.001$ \\
\hline$N F K B$ & $223(31-520)$ & $8(6-31)$ & $<0.001$ \\
\hline$H S D 11 B 1$ & $0.78(0.47-2.01)$ & $0.22(0.09-0.51)$ & $<0.001$ \\
\hline FOS & $0.112(0.03-0.19)$ & $0.006(0.01-0.04)$ & $<0.001$ \\
\hline$I K B K B$ & $111.2(54.5-299.3)$ & $21.2(5.4-52.4)$ & $<0.001$ \\
\hline CHUK & $115.3(20.5-223.6)$ & $5.8(2.28-12.91)$ & $<0.00$ \\
\hline \multicolumn{4}{|l|}{ Orbital fat } \\
\hline$N R 3 C 1$ & $118(53-171)$ & $108(46-349)$ & 1 \\
\hline$N F K B$ & $13.6(6.4-27.2)$ & $14.5(10.9-27.1)$ & 0.431 \\
\hline HSD11B1 & $1.17(0.59-2.63)$ & $1.56(0.55-5.21)$ & 0.44 \\
\hline FOS & $0.008(0.003-0.025)$ & $0.007(0.003-0.019)$ & 0.857 \\
\hline$I K B K B$ & $21.1(12.4-41.4)$ & $14.9(10.5-69.6)$ & 0.77 \\
\hline CHUK & $9.6(6.1-17.9)$ & $12.6(6.0-40.6)$ & 0.365 \\
\hline
\end{tabular}

GO, Graves' orbitophaty.

\section{Discussion}

We examined the mRNA expression levels of NR3C1, CHUK, IKBKB, FOS, NFKB and HSD11B1 in the EOM and $\mathrm{OF}$ tissue of GO patients during a clinically inactive period of the disease and at a distinct point of clinical inflammation in the classical model of GO based on the Rundel curve (32).

NR3C1, CHUK, IKBKB, FOS and NFKB were upregulated in the EOM of GO patients compared with controls. Although both values were elevated, the decreased relative expression of $N R 3 C 1 / N F K B$ ratio in EOM resulted from the increased NFKB expression observed in GO patients. This scenario might occur during the late phase of GO, during which a full inflammatory response could occur at the molecular level in association with the downregulation

Table 2 Relative NR3C1/NFKB and NR3C1/HSD11B1 expression rate in $\mathrm{GO}$ patients and controls. Data are presented as median (interquartile interval). Mann-Whitney test $\mathrm{GO}$ vs controls.

\begin{tabular}{|c|c|c|c|}
\hline & GO & Controls & $\boldsymbol{P}$ \\
\hline \multicolumn{4}{|l|}{ NR3C1/NFKB } \\
\hline $\begin{array}{l}\text { Muscle } \\
\text { tissue }\end{array}$ & $0.74(0.34-5.18)$ & $5.19(2.67-10.98)$ & 0.014 \\
\hline Orbital fat & $8.19(4.23-20.29)$ & 6.19 (3.16-21.36) & 0.523 \\
\hline \multicolumn{4}{|l|}{$\begin{array}{l}\text { NR3C1/ } \\
\quad \text { HSD11B1 }\end{array}$} \\
\hline $\begin{array}{l}\text { Muscle } \\
\text { tissue }\end{array}$ & $214.4(124.0-402.5)$ & $356.3(175.1-557.7)$ & 0.122 \\
\hline Orbital fat & 86.7 (64.1-121.5) & $64.5(42.3-120.6)$ & 0.229 \\
\hline
\end{tabular}


Table 3 NR3C1, NFKB, HSD11B1, FOS, IKBKB and CHUK expression in muscle and fat according to Nunery's classification of GO patients. Data are presented as median (interquartile interval).

\begin{tabular}{|c|c|c|c|}
\hline & Type $1(n=11)$ & Type $2(n=23)$ & $\boldsymbol{P}$ \\
\hline \multicolumn{4}{|l|}{ Muscle } \\
\hline$N R 3 C 1$ & $151(96-366)$ & 235 (95-446) & 0.552 \\
\hline$N F K B$ & $79(28-379)$ & $334(52-720)$ & 0.25 \\
\hline $\begin{array}{c}\text { NR3C1: } \\
\text { NFKB } \\
\text { ratio }\end{array}$ & $1.10(0.48-9.53)$ & $0.69(0.26-2.87)$ & 0.341 \\
\hline$H S D 11 B 1$ & $0.79(0.53-1.37)$ & $0.77(0.44-2.47)$ & 0.905 \\
\hline FOS & $0.045(0.02-0.11)$ & $0.14(0.07-0.2$ & 0.019 \\
\hline$I K B K B$ & $74.6(53.2-235.6)$ & $127.9(55.8-444.2)$ & 0.526 \\
\hline CHUK & $38.4(9.99-154.98)$ & $125.9(37.67-293.48)$ & 0.088 \\
\hline \multicolumn{4}{|l|}{ Orbital fat } \\
\hline NR3C1 & $118(42-479)$ & $112(58-157)$ & 0.985 \\
\hline$N F K B$ & $8(6-69)$ & $15(7-27)$ & 0.985 \\
\hline $\begin{array}{c}\text { NR3C1: } \\
\text { NFKB } \\
\text { ratio }\end{array}$ & $11.3(6.3-20.4)$ & $7.0(3.9-20.0)$ & 0.606 \\
\hline HSD11B1 & $0.95(0.58-6.44)$ & $1.18(0.62-2.12)$ & 0.804 \\
\hline FOS & $0.013(0.002-0.068)$ & $0.007(0.004-0.025)$ & 0.477 \\
\hline$I K B K B$ & 17.65 (10.93-90.03) & $21.34(12.74-40.67)$ & 0.894 \\
\hline CHUK & $6.72(5.20-34.52)$ & $10.46(7.16-17.13)$ & 0.633 \\
\hline
\end{tabular}

Mann-Whitney test $(P<0.05)$.

of NR3C1. Therefore, the glucocorticoid sensitivity might depend on the balance between NR3C1 expression and the concentration of inflammatory mediators such as NFKB and FOS.

The natural history of orbitopathy starts with an inflammatory phase that is characterized by classical phlogistic signs such as chemosis, hyperemia and pain, which are not always recognized by the patient. After this phase, orbital diseases tend to improve, with a regression of many symptoms as the fibrotic phase begins. The patients in the present analysis presented the fibrotic phase of disease, as suggested by a CAS score less than 2 and a lack of clinical activity.

The study by Tomilson et al. (28) reported an upregulation of IL-1 $\beta$, IL- 6 , TNF- $\alpha$ and other cytokines in adipose tissue from the orbit, indicating persistent, albeit clinically unapparent, inflammation. The elevated levels of $N F K B$ in EOM observed in our patients are in accordance with previous observations. Due to persistent local inflammation and elevated NFKB levels, NR3C1 levels increase to control or mitigate the inflammation, allowing greater binding of endogenous cortisol. Additionally, the local cortisol concentration increases as a consequence of the conversion of cortisone to cortisol mediated by the increased local activity of HSD11B1. Regarding fat tissue, our results differed from those reported by Tomlinson.
The GO group in the present analysis was predominantly myogenic (67.6\%) (i.e., subtype 2), with few cases classified as lipogenic (subtype 1). Unexpectedly, the expression ratio of $N R 3 C 1 / N F K B$ did not differ in relation to the Nunery classification subtypes, although a difference in c-Fos expression was detected.

The persistent inflammation that occurs in GO could be explained by an imbalance in the crosstalk between the NR3C1 and the NFKB pathway. The expression of NR3C1 and HSD11B1 increased, but NFKB activation predominated, resulting in a lower NR3C1:NFKB ratio in GO patients. The anti-inflammatory function of NR3C1 could be reduced in the orbital compartment, which was filled with differentiated fibroblasts and, in particular, in the inflammatory environment consisting of elevated levels of profibrotic cytokines (33).

NFKB activation involves diverse mechanisms. Inflammatory cytokines, such as TNF- $\alpha$, stimulate NFKB (canonical pathway) by modulating the IKK complex, generating phosphorylated IKBKB and allowing NFKB to dimerize, translocate to the nucleus and regulate inflammatory gene transcription. This process perpetuates the inflammation via the persistent production of cytokines (IL-6, TNF- $\alpha$ and IL-1 $\beta$ ) and chemokines (RANTES, IL-8) $(22,23,34)$.

Orbital fibroblasts expressing CD40 and CD4+ T cells expressing CD154 (CD40 ligand) bridge and interact, similar to the costimulation that occurs between an antigen-presenting cell and a $\mathrm{T}$ cell (35). This bridge (CD40-CD154) stimulates orbital fibroblasts to proliferate and produce additional IL- 1 and Il- $6(36,37)$ and induces the secretion of additional interferon- $\gamma$ and TNF- $\alpha$ by T cells, eventually resulting in the elevated production of hyaluronan and prostaglandin E2, which contribute to the development of orbital disease (15).

The alternative pathway (non-canonical pathway) of NFKB activation is initiated by the CD40 ligand on $\mathrm{T}$ cells $(37,38)$. Thus, we assume that the IKK-NFKB complex, through the canonical and non-canonical pathways, mediates fibroblast proliferation, activation and differentiation, and we have shown that FOS mRNA is highly expressed in the muscle of GO patients with the myogenic disease subtype. In the inflammatory pathway, FOS exists primarily in the FOS/Jun dimer, regulating growth factors, apoptosis, cell proliferation and differentiation (39).

At present, we cannot offer an adequate explanation for the absence of inflammatory mediators in the OF from GO patients. One possible mechanism is that our findings are representative of a select group of patients 
GO:

Graves' ophtalmopathy

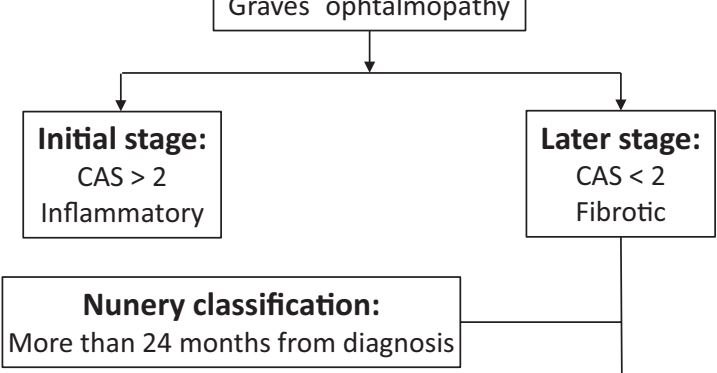

\section{Figure 1}

The role of inflammatory gene expression on late phase of GO.

representing a specific moment in the time line of GO at which orbital decompression can be performed. Our patients predominantly represented the myogenic type (subtype 2) of GO, in which orbital fibroblasts develop an exaggerated reaction compared with adipocytes (14), as identified by the elevated expression of $C H U K$, $I K B K B, N F k B$ and $F O S$, similar to other autoimmune diseases.

OF can initially exhibit robust inflammation in response to fibroblast differentiation; however, during the later stages of $\mathrm{GO}$, when adipocytes are smaller and less differentiated (40), adipogenesis is likely to be less intense, with reduced HSD11B1 activity (although HSD11B1 remains overexpressed), lower cortisol bioavailability and elevated expression of the profibrotic cytokines TGF- $\beta$, IL- 6 , TNF- $\alpha$ and IL- $1 \beta(36,41)$. In this context, orbital inflammation is perpetuated by increased expression levels of IKK-NFKB, FOS, TNF- $\alpha$ and IL-1 $\beta$ acting in a paracrine and autocrine manner to regulate NFKB $(42,43)$.

In the present study, during the late phase of disease, in which myofibroblasts predominate, profibrotic cytokines inhibit adipogenesis but perpetuate molecular inflammation, inducing increased expression of $C H U K$, $I K B K B, N F K B$ and FOS, and the crosstalk with GR leads to a phase that is less responsive to glucocorticoids.

An important limitation of our study was the lack of TRAb values of some patients in particular for patients who underwent radioiodine therapy when elevation of TRAb may worsen inflammation of the orbit and may influence the expression of inflammatory genes such as those studied in our work. Another important limitation is that RNA studies does not necessarily reflect the protein expression, but we believe that this molecular study brings new information about fibroblast behavior in the fibrotic phase of GO.

We have demonstrated that the CHUK-NFKB pathway and FOS are the most important mediators of the inflammatory response involved in GO (Fig. 1). Therefore, glucocorticoids and other drugs should not be delayed under any circumstance because they constitute the best treatment for GO. Another potential implication of our results relates to the use of glucocorticoid prophylaxis at the new onset or activation of GO in Graves' thyrotoxicosis after radioiodine therapy; newonset GO occurs in up to $15 \%$ of patients with Graves' disease despite the absence of any signs of GO after radioiodine treatment (44). Our observation of chronic inflammation of the orbit may be involved in new-onset GO after radioiodine treatment or in active GO during periods that are expected to exhibit less inflammatory activity, particularly the fibrotic phase.

Declaration of interest

The authors declare that there is no conflict of interest that could be perceived as prejudicing the impartiality of the research reported.

Funding

This work was supported by FAPESP grant 2012/04487-9.

\section{References}

1 Burch HB \& Wartofsky L. Graves' ophthalmopathy: current concepts regarding pathogenesis and management. Endocrine Reviews 199314 747-793. (doi:10.1210/edrv-14-6-747)

2 Baldeschi L, Wakelkamp IM, Lindeboom R, Prummel MF \& Wiersinga WM. Early versus late orbital decompression in Graves' orbitopathy: a retrospective study in 125 patients. Ophthalmology 2006113 874-878. (doi:10.1016/j.ophtha.2005.10.060)

3 Wiersinga WM \& Bartalena L. Epidemiology and prevention of Graves' ophthalmopathy. Thyroid 200212 855-860. (doi:10.1089/105 072502761016476) 
4 Bahn RS \& Heufelder AE. Pathogenesis of Graves' ophthalmopathy. New England Journal of Medicine 1993329 1468-1475. (doi:10.1056/ NEJM199311113292007)

5 Pappa A, Calder V, Ajjan R, Fells P, Ludgate M, Weetman AP \& Lightman S. Analysis of extraocular muscle-infiltrating $\mathrm{T}$ cells in thyroid-associated ophthalmopathy (TAO). Clinical \& Experimental Immunology 1997109 362-369. (doi:10.1046/j.13652249.1997.4491347.x)

6 Yang D, Hiromatsu Y, Hoshino T, Inoue Y, Itoh K \& Nonaka K. Dominant infiltration of $\mathrm{T}(\mathrm{H}) 1$-type $\mathrm{CD} 4+\mathrm{T}$ cells at the retrobulbar space of patients with thyroid-associated ophthalmopathy. Thyroid 19999 305-310. (doi:10.1089/thy.1999.9.305)

7 Heufelder AE \& Bahn RS. Modulation of Graves' orbital fibroblast proliferation by cytokines and glucocorticoid receptor agonists. Investigative Ophthalmology \& Visual Science 199435 120-127.

8 Koumas L, Smith TJ \& Phipps RP. Fibroblast subsets in the human orbit: Thy-1+ and Thy-1- subpopulations exhibit distinct phenotypes. European Journal of Immunology 200232 477-485. (doi:10.1002/1521-4141(200202)32:2<477::AIDIMMU477>3.0.CO;2-U)

9 Smith TJ, Koumas L, Gagnon A, Bell A, Sempowski GD, Phipps RP $\&$ Sorisky A. Orbital fibroblast heterogeneity may determine the clinical presentation of thyroid-associated ophthalmopathy. Journal of Clinical Endocrinology and Metabolism 200287 385-392. (doi:10.1210/ jcem.87.1.8164)

10 Koumas L, Smith TJ, Feldon S, Blumberg N \& Phipps RP. Thy-1 expression in human fibroblast subsets defines myofibroblastic or lipofibroblastic phenotypes. American Journal of Pathology 2003163 1291-1300. (doi:10.1016/S0002-9440(10)63488-8)

11 Prabhakar BS, Bahn RS \& Smith TJ. Current perspective on the pathogenesis of Graves' disease and ophthalmopathy. Endocrine Reviews 200324 802-835. (doi:10.1210/er.2002-0020)

12 Feldon SE, O'Loughlin CW, Ray DM, Landskroner-Eiger S, Seweryniak KE \& Phipps RP. Activated human T lymphocytes express cyclooxygenase- 2 and produce proadipogenic prostaglandins that drive human orbital fibroblast differentiation to adipocytes. American Journal of Pathology 2006169 1183-1193. (doi:10.2353/ajpath.2006.060434)

13 Lehmann GM, Feldon SE, Smith TJ \& Phipps RP. Immune mechanisms in thyroid eye disease. Thyroid 200818 959-965. (doi:10.1089/thy.2007.0407)

14 Smith TJ, Tsai CC, Shih MJ, Tsui S, Chen B, Han R, Naik V, King CS, Press C, Kamat $\mathrm{S}$ et al.Unique attributes of orbital fibroblasts and global alterations in IGF-1 receptor signaling could explain thyroidassociated ophthalmopathy. Thyroid 200818 983-988. (doi:10.1089/ thy.2007.0404)

15 Bahn RS. Graves' ophthalmopathy. New England Journal of Medicine 2010362 726-738. (doi:10.1056/NEJMra0905750)

16 Aniszewski JP, Valyasevi RW \& Bahn RS. Relationship between disease duration and predominant orbital T cell subset in Graves' ophthalmopathy. Journal of Clinical Endocrinology and Metabolism 2000 85 776-780. (doi:10.1210/jc.85.2.776)

17 Shen J, Li Z, Li W, Ge Y, Xie M, Lv M, Fan Y, Chen Z, Zhao D \& Han Y. Th1, Th2, and Th17 cytokine involvement in thyroid associated ophthalmopathy. Disease Markers 20152015609593. (doi:10.1155/2015/609593)

18 Fang S, Huang Y, Wang S, Zhang Y, Luo X, Liu L, Zhong S, Liu X, Li D, Liang R et al. IL-17A exacerbates fibrosis by promoting the proinflammatory and profibrotic function of orbital fibroblasts in TAO. Journal of Clinical Endocrinology and Metabolism 2016101 2955-2965. (doi:10.1210/jc.2016-1882)

19 Hoffmann A, Leung TH \& Baltimore D. Genetic analysis of NF-kB/ Rel transcription factors defines functional specificities. EMBO Journal 200322 5530-5539. (doi:10.1093/emboj/cdg534)

20 Hess J, Angel P \& Schorpp-Kistner M. AP-1 subunits: quarrel and harmony among siblings. Journal of Cell Science 2004117 5965-5973. (doi:10.1242/jcs.01589)
21 Ahmad R, Raina D, Trivedi V, Ren J, Rajabi H, Kharbanda S \& Kufe D. MUC1 oncoprotein activates the IkappaB kinase beta complex and constitutive NF-kappaB signalling. Nature Cell Biology 20079 1419-1427. (doi:10.1038/ncb1661)

22 Hayden MS \& Ghosh S. Signaling to NF-кB. Genes \& Development 2004 18 2195-2224. (doi:10.1101/gad.1228704)

23 Hayden MS \& Ghosh S. Shared principles in NF-kappaB signaling. Cell 2008132 344-362. (doi:10.1016/j.cell.2008.01.020)

24 Gilmore TD. Introduction to NF-kappaB: players, pathways, perspectives. Oncogene 200625 6680-6684. (doi:10.1038/ sj.onc.1209954)

25 Verstrepen L, Bekaert T, Chau TL, Tavernier J, Chariot A \& Beyaert R. TLR-4, IL-1R and TNF-R signaling to NF-kB: variations on a common theme. Cellular and Molecular Life Sciences 200865 2964-2978. (doi:10.1007/s00018-008-8064-8)

26 Northrop JP, Crabtree GR \& Mattila PS. Negative regulation of interleukin 2 transcription by the glucocorticoid receptor. Journal of Experimental Medicine 1992175 1235-1245. (doi:10.1084/ jem.175.5.1235)

27 Chikanza LC \& Panayi GS. The effects of hydrocortisone on in vitro lymphocyte proliferation and interleukin-2 and -4 production in corticosteroid sensitive and resistant subjects. European Journal of Clinical Investigation 199323 845-850. (doi:10.1111/j.1365-2362.1993.tb00740.x)

28 Tomlinson JW, Durrani OM, Bujalska IJ, Gathercole LL, Tomlins PJ, Reuser TT, Rose GE, Curnow SJ, Stewart PM, Walker EA et al. The role of 11beta-hydroxysteroid dehydrogenase 1 in adipogenesis in thyroid-associated ophthalmopathy. Journal of Clinical Endocrinology and Metabolism 201095 398-406. (doi:10.1210/jc.2009-0873)

29 Bartley GB \& Gorman CA. Diagnostic criteria for Graves' ophthalmopathy. American Journal of Ophthalmology 1995119 792-795. (doi:10.1016/S0002-9394(14)72787-4)

30 Mourits MP, Koornneef L, Wiersinga WM, Prummel MF, Berghout A \& van der Gaag R. Clinical criteria for the assessment of disease activity in Graves' ophthalmopathy: a novel approach. British Journal of Ophthalmology 198973 639-644. (doi:10.1136/bjo.73.8.639)

31 Werner SC. Classification of the eye changes of Grave's disease. Journal of Clinical Endocrinology and Metabolism 196929 982-984. (doi:10.1210/jcem-29-7-982)

32 Bartley GB. Rundle and his curve. Archives of Ophthalmology 2011129 356-358. (doi:10.1001/archophthalmol.2011.29)

33 Khong JJ, McNab AA, Ebeling PR, Craig JE \& Selva D. Pathogenesis of thyroid eye disease: review and update on molecular mechanisms. British Journal of Ophthalmology 2016100 142-150. (doi:10.1136/ bjophthalmol-2015-307399)

34 Tian B, Nowak DE \& Brasier AR. A TNF-induced gene expression program under oscillatory NF-kappaB control. BMC Genomics 20056 137. (doi:10.1186/1471-2164-6-137)

35 Grewal IS \& Flavell RA. The role of CD40 ligand in costimulation and T-cell activation. Immunological Reviews 1996153 85-106. (doi:10.1111/j.1600-065X.1996.tb00921.x)

36 Feldon SE, Park DJ, O'Loughlin CW, Nguyen VT, Landskroner-Eiger S, Chang D, Thatcher TH \& Phipps RP. Autologous T-lymphocytes stimulate proliferation of orbital fibroblasts derived from patients with Graves' ophthalmopathy. Investigative Ophthalmology \& Visual Science 200546 3913-3921. (doi:10.1167/iovs.05-0605)

37 Senftleben U, Cao Y, Xiao G, Greten FR, Krahn G, Bonizzi G, Chen Y, $\mathrm{Hu} \mathrm{Y}$, Fong A, Sun SC et al. Activation by IKKalpha of a second, evolutionary conserved, NF-kappa B signaling pathway. Science 2001 293 1495-1499. (doi:10.1126/science.1062677)

38 Pal S, Bhattacharjee A, Ali A, Mandal NC, Mandal SC \& Pal M. Chronic inflammation and cancer: potential chemoprevention through nuclear factor kappa B and p53 mutual antagonism. Journal of Inflammation 201411 23. (doi:10.1186/1476-9255-11-23)

39 Zenz R, Eferl R, Scheinecker C, Redlich K, Smolen J, Schonthaler HB, Kenner L, Tschachler E \& Wagner EF. Activator protein 1 (Fos/Jun) 
functions in inflammatory bone and skin disease. Arthritis Research \& Therapy 200810 201. (doi:10.1186/ar2338)

40 Bujalska IJ, Durrani OM, Abbott J, Onyimba CU, Khosla P, Moosavi AH, Reuser TT, Stewart PM, Tomlinson JW, Walker EA et al. Characterisation of 11beta-hydroxysteroid dehydrogenase 1 in human orbital adipose tissue: a comparison with subcutaneous and omental fat. Journal of Endocrinology 2007192 279-288. (doi:10.1677/ JOE-06-0042)

41 Han R \& Smith TJ. Induction by IL-1 beta of tissue inhibitor of metalloproteinase-1 in human orbital fibroblasts: modulation of gene promoter activity by IL-4 and IFN-gamma.
Journal of Immunology 2005174 3072-3079. (doi:10.4049/ jimmunol.174.5.3072)

42 Ghosh S \& Karin M. Missing pieces in the NF-kappaB puzzle. Cell 2002 109 (Supplement) S81-S96. (doi:10.1016/S0092-8674(02)00703-1)

43 Medzhitov R. Toll-like receptors and innate immunity. Nature Reviews: Immunology 20011 135-145. (doi:10.1038/35100529)

44 Vannucchi G, Campi I, Covelli D, Dazzi D, Curro N, Simonetta S, Ratiglia R, Beck-Peccoz P \& Salvi M. Graves' orbitopathy activation after radioactive iodine therapy with and without steroid prophylaxis. Journal of Clinical Endocrinology and Metabolism 200994 3381-3386. (doi:10.1210/jc.2009-0506)

Received 18 November 2016

Revised version received 20 December 2016

Accepted 11 January 2017 